\title{
NOTE ON AN ELEMENTARY GEOMETRIC EXISTENCE THEOREM
}

BY P. W. KETCHUM

1. Introduction. By the $\prod$-moment of a set of points $c_{1}, c_{2}, \cdots, c_{m},(m>1)$, with respect to a particular $c_{q}$ we mean the product of the $m-1$ distances from $c_{q}$ to the other $c$ 's. The object of the present note is to prove the following proposition:

THEOREM 1. For any given set of distinct points $a_{1}, a_{2}, \cdots, a_{n}$, $(n>1)$, in the plane, there will exist a set of points $b_{1}, b_{2}, \cdots, b_{n}$, distinct from the a's, such that the П-moment of the a's and b's with respect to $a_{i}$ is the same for all $i$.

This theorem is obviously a special case of the following more general theorem:

Theorem 2. Let $a_{1}, a_{2}, \cdots, a_{n},(n>1)$, be a set of distinct points in a plane and let $d_{i, j}$ be the distance from $a_{i}$ to $a_{j},(i \neq j) .{ }^{*}$ Let $r_{i}$ be any given ray (half line) issuing from $a_{i}$. Then for every sufficiently small positive number $\delta$, say for $\delta \leqq \delta_{0}$, there exists a set of points $b_{1}, b_{2}, \cdots, b_{n}$ satisfying the two conditions (a) the point $b_{i}$ is on the ray $r_{i}$, and (b) if $D_{i, j}$ is the distance from $a_{i}$ to $b_{j}$, then

$$
d_{i, 1} d_{i, 2} \cdots d_{i, i-1} d_{i, i+1} \cdots d_{i, n} D_{i, 1} D_{i, 2} \cdots D_{i, n}=\delta
$$

for all values of $i$.

2. An Outline of the Procedure. An algebraic proof of the existence of the desired solutions of (1) seems to be difficult, so we prefer to use the method of successive approximations. The formal details of our procedure are a little tedious, but the underlying idea is relatively simple, namely: Since $\delta$ is small, the point $b_{i}$ will be much closer to $a_{i}$ then to any of the other $a$ 's or $b$ 's. Thus, if $b_{i}$ is shifted slightly, the quantity $D_{i, i}$ will change relatively much more than any of the other distances. This has the effect of making the product, $\Pi$, in (1) approximately a

* Throughout this note, $i$ and $j$ will range over the values $1,2,3, \cdots, n$, and $k$ over the values $1,2,3, \cdots$. 
function of one variable $D_{i, i}$ only. As a first approximation we therefore assume that $D_{i, j}=d_{i, j}$ for $i \neq j$, and calculate $D_{i, i}$ so that the product $\prod$ will equal $\delta$. This will determine new distances $D_{i, j}, i \neq j$, slightly different from $d_{i, j}$, which one may use as a second approximation for the calculation of $D_{i, i}$; and so on. It is intuitively evident that the process will be a convergent one. We now proceed to the details.

3. Proof of Theorem 2. We are going to prove the theorem for the particular value

$\delta_{0}=n^{-1} 2^{-3 n} d^{3 n-2} D^{1-n}, \quad$ where $D=\max \left(d_{i, j}\right), \quad d=\min \left(d_{i, j}\right)$.

Put $D_{i, j}^{(0)}=d_{i, j}$ for $i \neq j$, and let $D_{i, j}^{(k)}$ be a set of numbers satisfying the relations

$$
D_{i, i}^{(k)}=\frac{\delta}{\prod_{j}^{(i)}\left(d_{i, j} D_{i, j}^{(k-1)}\right)} .
$$

Here $\prod_{j}{ }^{(i)}$ is a symbol indicating a product to be taken over all values of $j$ with the exception of the value $j=i$, which is to be omitted. Denote by $b_{i}{ }^{(k)}$ the point on $r_{i}$ at distance $D_{i, i}^{(k)}$ from $a_{i}$. Let $D_{i, j}^{(k)}$ be the distance from $a_{i}$ to $b_{j}{ }^{(k)}$. The quantities $D_{i, j}^{(k)}$ and $b_{i}{ }^{(k)}$ are thus uniquely defined by recurrence.

We first show that the points $b_{j}{ }^{(k)}$ have for each $j$ a unique limiting point as $k \rightarrow \infty$. Set

$$
\eta_{i, i}^{(1)}=D_{i, i}^{(1)}, \quad \eta_{i, j}^{(1)}=D_{i, j}^{(1)}-D_{i, j}^{(0)} \text { for } i \neq j,
$$

and

$$
\eta_{i, j}^{(k+1)}=D_{i, j}^{(k+1)}-D_{i, j}^{(k)} .
$$

It will then be sufficient to prove that

$$
\left|\eta_{i, i}^{(k+1)}\right|<n^{-1} 2^{2-5 n-3 k} d^{n} D^{1-n},
$$

since the right-hand member is the $k$ th term of a convergent geometric series. We proceed to prove (3) by induction. Put

$$
\begin{gathered}
\eta_{k}=\max \left|\eta_{i, i}^{(k)}\right|=\max \left|\eta_{i, j}^{(k)}\right|, \\
D_{0}=D, \quad D_{k}=D_{k-1}+\eta_{k}, \quad d_{0}=d, \quad d_{k}=d_{k-1}-\eta_{k} .
\end{gathered}
$$


Then

$$
D_{i, j}^{(k)} \leqq D_{k}, \quad D_{i, j}^{(k)} \geqq d_{k} .
$$

Also

$$
\left|\eta_{i, i}^{(k+1)}\right|=\frac{\delta\left|\prod_{j}^{(i)} D_{i, j}^{(k)}-\prod_{j}^{(i)} D_{i, j}^{(k-1)}\right|}{\prod_{j}^{(i)}\left|d_{i, j} D_{i, j}^{(k-1)} D_{i, j}^{(k)}\right|}
$$

Calling the numerator in this expression $\delta K_{i}{ }^{(k+1)}$ and the denominator $T_{i}{ }^{(k+1)}$, we have

$$
\begin{aligned}
K_{i}^{(k+1)} & =\left|\prod_{j}^{(i)}\left(D_{i, j}^{(k-1)}+\eta_{i, j}^{(k)}\right)-\prod_{j}^{(i)} D_{i, j}^{(k-1)}\right| \\
& \leqq(n-1) \eta_{k} D_{k-1}^{n-2}+C_{n-1,2} \eta_{k}^{2} D_{k-1}^{n-3}+\cdots+\eta_{k}^{n-1},
\end{aligned}
$$

where the $C$ 's are binomial coefficients; and

$$
T_{i}^{(k+1)} \geqq d^{n-1} d_{k-1}^{n-1} d_{k}^{n-1},
$$

provided $d_{k}$ is positive for all $k$. Now from (2) and our hypothesis on $\delta$,

$$
\left|\eta_{i, i}^{(1)}\right| \leqq n^{-1} 2^{-3 n} d^{n} D^{1-n}
$$

Hence

$$
\eta_{1} \leqq n^{-1} 2^{-3 n} d^{n-1} D^{1-n} D_{0} .
$$

An easy computation shows that

$$
K_{i}^{(2)}<n^{-1} 2^{-3 n} d^{n-1} D^{1-n} D_{0}^{n-1}[(n-1)+1]=2^{-3 n} d^{n-1} .
$$

Now

$$
d_{1}=d-\eta_{1}>d / 2, \quad T_{i}^{(2)}>2^{1-n} d^{3 n-3}
$$

and

$$
\left|\eta_{i, i}^{(2)}\right|=\frac{\delta K_{i}^{(2)}}{T_{i}(2)}<n^{-1} 2^{-1-5 n} D^{1-n} d^{n} .
$$

Thus (3) is seen to be satisfied for the first value of $k$, namely $k=1$. 
Next we assume that (3) is satisfied for $k=1,2, \cdots, \mu-1$. Then

$$
\eta_{\mu} \leqq n^{-1} 2^{5-5 n-3 \mu} d^{n-1} D^{1-n} D_{\mu-1} .
$$

An easy computation shows that

$$
K_{i}^{(\mu+1)} \leqq n^{-1} 2^{5-5 n-3 \mu} d^{n-1} D^{1-n} D_{\mu-1}^{n-1}[(n-1)+1] .
$$

Now

$$
\begin{aligned}
D_{\mu} & =D+\eta_{1}+\eta_{2}+\cdots+\eta_{\mu} \\
& <D\left[1+\frac{1}{2}+\frac{1}{4}+\cdots\right]=2 D,
\end{aligned}
$$

from our assumption that (3) holds for $k=1,2, \cdots, \mu-1$. Hence

$$
K_{i}^{(\mu-1)}<2^{4-4 n-3 \mu} d^{n-1} .
$$

Also

$$
\begin{aligned}
d_{\mu} & =d-\eta_{1}-\eta_{2}-\cdots-\eta_{\mu} \\
& >d\left[1-\frac{1}{4}-\frac{1}{8}-\frac{1}{16}-\cdots\right]=d / 2 .
\end{aligned}
$$

Hence

$$
T_{i}^{(\mu+1)}>2^{2-2 n} d^{3 n-3}
$$

and

$$
\left|\eta_{i, i}^{(\mu+1)}\right|=\frac{\delta K_{i}^{(\mu+1)}}{T_{i}^{(\mu+1)}}<n^{-1} 2^{2-5 n-3 \mu} d^{n} D^{1-n},
$$

so that (3) is true when $k=\mu$. The induction is thus complete and (3) is proved, and the points $b_{j}{ }^{(k)}$ have a unique limit point.

Now take the points $b_{j}$ of Theorem II as the limiting points of $b_{j}^{(k)}$. Let

Since

$$
\delta_{i}^{(k)}=\prod_{j}^{(i)} d_{i, j} \prod_{j} D_{i, j}^{(k)}
$$

$$
D_{i, j}=\lim _{k \rightarrow \infty} D_{i, j}^{(k)}
$$


we have

$$
\lim _{k \rightarrow \infty} \delta_{i}^{(k)}=\prod_{j}^{(i)} d_{i, j} \prod_{j} D_{i, j}
$$

Now

$$
\left|\delta_{i}^{(k)}-\delta\right|=\delta\left|1-\frac{\prod_{j}^{(i)} D_{i, j}^{(k)}}{\prod_{j}^{(i)} D_{i, j}^{(k-1)}}\right|
$$

But the quantity on the right approaches zero, so that $\delta_{i}{ }^{(k)} \rightarrow \delta$ as $k \rightarrow \infty$. We thus have (1), and the theorem is proved.

Institute for Advanced Study

\title{
AN INVOLUTORIAL LINE TRANSFORMATION IN $S_{4}$
}

\author{
BY C. R. WYLIE, JR.
}

1. Introduction. It is a well known fact that all planes which meet four general lines of $S_{4}{ }^{*}$ are met by a fifth line. The remarkable configuration determined by five such "associated lines" is discussed in a number of places in the literature. $\dagger$ In the present paper an involutorial line transformation suggested by the figure of five associated lines is discussed, both as a line involution in $S_{4}$, and as a point involution on a certain $V_{6}{ }^{5}$ in $S_{9}$. In $\S \S 2-6$ the involution is treated at some length by purely synthetic methods. The final section $(\$ 7)$ contains a brief analytic treatment, including the equations of the involution, and the equations of the invariant and singular elements. The involu-

* We shall use the conventional symbol $S_{m}$ to indicate a linear space of dimension $m$. A variety of order $r$ and of dimension $m$ we shall designate by the symbol $V_{m}{ }^{r}$.

$\dagger$ Welchman, W. G., Plane congruences of the second order in space of four dimensions and fifth incidence theorems, Proceedings of the Cambridge Philosophical Society, vol. 28 (1931-1932), pp. 275-284.

Baker, H. F., On a proof of the theorem of a double six of lines by projection from four dimensions, Proceedings of the Cambridge Philosophical Society, vol. 20 (1920-1921), pp. 133-144.

Baker, H. F., Principles of Geometry, Cambridge University Press, 1925, vol. IV, Chapter V. 